\title{
Study of the stabilization energies of halide-water clusters: An application of first-principles interaction potentials based on a polarizable and flexible model
}

\author{
Regla Ayala, José M. Martínez, Rafael R. Pappalardo, and Enrique Sánchez Marcos ${ }^{\text {a) }}$ \\ Departamento de Química Física, Universidad de Sevilla, 41012 Sevilla, Spain
}

(Received 5 April 2004; accepted 12 July 2004)

\begin{abstract}
The aim of this work is to compute the stabilization energy $E_{\text {stab }}(n)$ of $\left[X\left(\mathrm{H}_{2} \mathrm{O}\right)_{n}\right]^{-}(X \equiv \mathrm{F}, \mathrm{Br}$, and I for $n=1-60$ ) clusters from Monte Carlo simulations using first-principles $a b$ initio potentials. Stabilization energy of $\left[X\left(\mathrm{H}_{2} \mathrm{O}\right)_{n}\right]^{-}$clusters is defined as the difference between the vertical photodeachment energy of the cluster and the electron affinity of the isolated halide. On one hand, a study about the relation between cluster structure and the $E_{\text {stab }}(n)$ value, as well as the dependence of the latter with temperature is performed, on the other hand, a test on the reliability of our recently developed first-principles halide ion-water interaction potentials is carried out. Two different approximations were applied: (1) the Koopmans' theorem and (2) calculation of the difference between the interaction energy of $\left[X\left(\mathrm{H}_{2} \mathrm{O}\right)_{n}\right]^{-}$and $\left[X\left(\mathrm{H}_{2} \mathrm{O}\right)_{n}\right]$ clusters using the same ab initio interaction potentials. The developed methodology allows for using the same interaction potentials in the case of the ionic and neutral clusters with the proviso that the charge of the halide anion was switched off in the latter. That is, no specific parametrization of the interaction potentials to fit the magnitude under study was done. The good agreement between our predicted $E_{\text {stab }}(n)$ and experimental data allows us to validate the first-principles interaction potentials developed elsewhere and used in this study, and supports the fact that this magnitude is mainly determined by electrostatic factors, which can be described by our interaction potentials. No relation between the value of $E_{\text {stab }}(n)$ and the structure of clusters has been found. The diversity of $E_{\text {stab }}(n)$ values found for different clusters with similar interaction energy indicates the need for statistical information to properly estimate the stabilization energy of the halide anions. The effect of temperature in the prediction of the $E_{\text {stab }}(n)$ is not significant as long as it was high enough to avoid cluster trapping into local equilibrium configurations which guarantees an appropriate sampling of the configurational space. Parallel tempering method was applied in particular cases to guarantee satisfactory sampling of clusters at low temperature. (C) 2004 American Institute of Physics.
\end{abstract}

[DOI: 10.1063/1.1788660]

\section{INTRODUCTION}

Experimental and theoretical investigations performed on ion-solvent clusters are of great importance to help in understanding the solvation phenomena of ionic solutions. ${ }^{1,2}$ The available experimental techniques, such as photoelectron spectrometry, allow for the study of a cluster with a specific size. On many occasions, the analysis of the results of these observations needs the knowledge of the structure of the clusters considered. In this sense, computer simulations have emerged as a powerful tool for investigating the microscopic properties of clusters in gas phase and liquid solutions., Nevertheless, the reliability of the results derived from numerical simulations depends critically on the potential model describing the interactions among the particles defining the system.

In our previous halide solvation studies ${ }^{5,6}$ the development of first-principles halide ion-water interaction potentials using the MCDHO (Ref. 7) (mobile charge densities in harmonic oscillator) model potential was presented. This

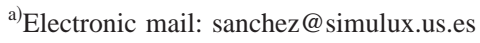

model includes the polarizable and flexible character of the interacting particles that form the system. Our results ${ }^{5,6,8}$ indicated that the halide ion-water interaction potentials for $\mathrm{F}^{-}, \mathrm{Br}^{-}$, and $\mathrm{I}^{-}$anions are able to provide structural and thermodynamic properties of both halide solutions and gas phase clusters in good agreement with the experimental estimations.

The study here presented focuses on the stabilization energy $\left(E_{\text {stab }}\right)$ of halide-water clusters derived from photoelectron spectroscopy (PES). PES is well established in studying the electronic structure of negatively charged clusters. ${ }^{9-15}$ The aim of this work is twofold. First, the relation between cluster structure and stabilization energy, as well as the dependence of the latter with temperature are studied. Second, a test on our first-principles halide ion-water interaction potentials developed and published elsewhere ${ }^{5,6}$ is carried out, by proposing the estimation of $E_{\text {stab }}$ from the developed potentials.

$E_{\text {stab }}$ is defined as the energy gap between the vertical photodetachment energy of the ion in the cluster $B E_{v}$ and the electron affinity of the bare ion $E A$, 


$$
E_{\text {stab }}(n)=B E_{v}(n)-E A,
$$

$n$ being the number of solvent molecules in the cluster.

Markovich et al. ${ }^{14,15}$ present the experimental photoelectron spectra of $\mathrm{Cl}^{-}, \mathrm{Br}^{-}$, and $\mathrm{I}^{-}$hydrated clusters considering up to $n=60$ water molecules in the case of the iodide anion. Wang and co-workers ${ }^{16}$ present experimental $E_{\text {stab }}$ up to $n=4$ for $\mathrm{F}^{-}$water clusters. Larger clusters of the fluoride anion could not be studied because of their small detachment cross section and extremely high electron binding energies.

Several theoretical studies about the stabilization energy of the halide-water clusters are available in literature. These studies can be divided into two different groups depending on the origin of the cluster geometries.

The first group is formed by those works that use a set of aggregates derived from $a b$ initio calculations to obtain the stabilization energies of the $\left[X\left(\mathrm{H}_{2} \mathrm{O}\right)_{n}\right]^{-}$clusters. Kim and co-workers ${ }^{17-21}$ work, among other properties, on the $a b$ initio prediction of the stabilization energies of $\left[X\left(\mathrm{H}_{2} \mathrm{O}\right)_{n}\right]^{-}$ clusters $(X \equiv \mathrm{F}, \mathrm{Cl}, \mathrm{Br}$, and $\mathrm{I}, n=1-6)$ by both calculating the interaction energies difference between the ionic and the neutral cluster and using the Koopmans' theorem. ${ }^{22}$ Combariza $e t a l .^{23-25}$ carry out an $a b$ initio study of the chloride-, bromide-, and iodide-water clusters to establish which are the prevalent isomers in gas phase. They used the agreement between stabilization energies obtained from the Koopman's theorem and binding enthalpies with the experimental data.

The second group is formed by the studies that compute stabilization energies of $\left[X\left(\mathrm{H}_{2} \mathrm{O}\right)_{n}\right]^{-}$clusters using structures from numerical simulations. Dang et al. ${ }^{26,27}$ study the iodide ion-water system using additive and nonadditive model interaction potentials, as well as classical and quantum mechanical simulations. Two different interaction potentials were considered for the computation of the interaction energies of ionic and neutral halide-water clusters. For the latter, the interaction potential was fitted in such way that the experimental $E_{\text {stab }}(1)$ was properly reproduced. These authors conclude that there is a small effect with respect to both the temperature and the inclusion of quantum effects to reproduce experimental stabilization energy. $E_{\text {stab }}$ depends on the differences between neutral and ionic ground states and, even though quantum effects are important in both states, there is a cancellation of these quantum effects when the difference is carried out. Perera et al. ${ }^{28,29}$ suggest the need for using a halide ion-water interaction potential including many-body effects to reproduce their experimental stabilization energies. These authors find that the temperature at which numerical simulation is carried out does not have influence on $E_{\text {stab }}$, as long as the configurational space is properly sampled. As Dang et al., ${ }^{26,27}$ Perera et al. ${ }^{28,29}$ consider different interaction potentials for computing the interaction energy of the ionic and neutral halide-water aggregates.

To our best knowledge, there are no $E_{\text {stab }}$ estimations derived only from first-principles halide ion-water interaction potentials.

\section{COMPUTATIONAL METHODS}

The quantum-mechanical computation of the photodetachment energy for a cluster with $n$ solvent molecules re- quires the determination of the energy associated to the electron ionization process for the halide anion and the solvated one. Alternatively, the photodetachment energy could be calculated by switching off the interactions related to the negative charge on the anion, or removing an electron from the highest occupied molecular orbital in theoretical calculations. It is worth pointing out that when $n$ increases, statistical distributions become important and single point calculations on a given structure might be not longer representative. In addition, for medium and large values of $n$ ab initio computations are not feasible because of cost.

There is experimental uncertainty in the temperature of experiments which were obtained possibly around $\sim 70 \mathrm{~K} .{ }^{14,15}$ To establish the importance of temperature effects on the cluster structure, Monte Carlo (MC) simulations of $\left[X\left(\mathrm{H}_{2} \mathrm{O}\right)_{n}\right]^{-}$clusters $(X \equiv \mathrm{F}, \mathrm{Br}$, and $\mathrm{I})$ at $70,150,200$, and $250 \mathrm{~K}$ were carried. For each cluster $200 \mathrm{M}$ configurations $\left(M \equiv 10^{6}\right)$ of $\mathrm{MC}$ simulations were produced. Configurations were saved every $1 M$ steps for further analysis.

In this work, stabilization energies have been obtained from two different approximations employing configurations from the MC simulations. These two approximations are:

(1) Using the Koopmans' theorem. ${ }^{22}$ Although some authors ${ }^{17,20,21,24}$ demonstrate that the use of Koopmans' theorem to compute ionization potentials of bare anions and $\left[X\left(\mathrm{H}_{2} \mathrm{O}\right)_{n}\right]^{-}$clusters overestimates the results by $0.4-0.5$ $\mathrm{eV}$, its use to get ionization potential differences is suitable ${ }^{23-25}$ because almost the same error exists in both systems.

(2) Calculating the difference between the interaction energy of $\left[X\left(\mathrm{H}_{2} \mathrm{O}\right)_{n}\right]^{-}$and $\left[X\left(\mathrm{H}_{2} \mathrm{O}\right)_{n}\right]$ clusters using ab initio interaction potentials. Differing from the strategy followed by Dang et al. ${ }^{26,27}$ and Perera et al. ${ }^{28,29}$ we do not develop different interaction potentials for the neutral and ionic aggregates. This way, the interaction energy of the neutral cluster was obtained by switching off the halide charge of the MCDHO halide-water interaction potential while the rest of parameters were kept unchanged.

The theoretical ionization potentials (IP) by means of Koopmans' theorem were computed at the self-consistent field level considering DZ basis sets augmented by polarization and diffuse basis functions. ${ }^{30-34}$ An effective core pseudopotential (ECP) was used to describe the core electrons in the case of the iodide ion, namely, the SDB-ECP, ${ }^{35}$ while the aug-cc-pVTZ basis sets augmented with polarization functions ${ }^{36}$ were used for the valence electrons.

To compute the average $E_{\text {stab }}(n)$ in $\left[X\left(\mathrm{H}_{2} \mathrm{O}\right)_{n}\right]^{-}$clusters using the MCDHO ion-water interaction potentials two assumptions were made. First, the photodetachment process is fast enough so that the geometry of the cluster does not change. Second, the induced polarization on the water molecules in $\left[X\left(\mathrm{H}_{2} \mathrm{O}\right)_{n}\right]^{-}$remains unchanged in the neutral cluster, that is to say, it is assumed that neither nuclear nor electronic relaxation in the cluster take place during the photoionization process. These assumptions are, basically, the same as that in Koopmans' theorem.

The magnitudes on the right-hand side of Eq. (1) are defined as 
TABLE I. Comparison of predicted and experimental $E_{\text {estab }}(n)(\mathrm{eV})$ of $\left[X\left(\mathrm{H}_{2} \mathrm{O}\right)_{n}\right]^{-}$clusters at $200 \mathrm{~K}$. The uncertainty in the experimental values is $0.03 \mathrm{eV}$.

\begin{tabular}{lcccccccccc}
\hline \hline & \multicolumn{3}{c}{$\mathrm{F}^{-}$} & \multicolumn{3}{c}{$\mathrm{Br}^{-}$} & & \multicolumn{3}{c}{$\mathrm{I}^{-}$} \\
\cline { 2 - 10 }$n$ & \multirow{2}{*}{ MCDHO } & Koopmans & Expt. $^{\mathrm{a}}$ & MCDHO & Koopmans & Expt. $^{\mathrm{b}}$ & MCDHO & Koopmans & Expt. $^{\mathrm{c}}$ \\
\hline 1 & $1.74(0.08)^{\mathrm{d}}$ & $1.49(0.04)$ & 1.40 & $0.58(0.04)$ & $0.54(0.04)$ & 0.57 & $0.43(0.03)$ & $0.43(0.03)$ & 0.45 \\
2 & $2.95(0.08)$ & $2.40(0.06)$ & 2.39 & $1.11(0.06)$ & $1.03(0.05)$ & 1.08 & $0.79(0.05)$ & $0.81(0.07)$ & 0.86 \\
3 & $3.80(0.19)$ & $3.36(0.14)$ & 3.19 & $1.57(0.11)$ & $1.45(0.10)$ & 1.58 & $1.13(0.05)$ & $1.17(0.08)$ & 1.23 \\
4 & $4.52(0.17)$ & $3.88(0.10)$ & 3.95 & $1.95(0.10)$ & $1.79(0.10)$ & 1.91 & $1.40(0.08)$ & $1.45(0.10)$ & 1.53 \\
\hline \hline
\end{tabular}

${ }^{\mathrm{a}}$ Reference 16

${ }^{\mathrm{b}}$ Reference 42.

${ }^{\mathrm{c}}$ Reference 13.

${ }^{\mathrm{d}}$ Standard deviation in $\mathrm{eV}$.

$$
B E_{v}(n)=E_{n}-E_{n}^{-}
$$

and

$$
E A=E-E^{-},
$$

where $E_{n}, E_{n}^{-}, E$, and $E^{-}$are the total energies of $\left[X\left(\mathrm{H}_{2} \mathrm{O}\right)_{n}\right],\left[X\left(\mathrm{H}_{2} \mathrm{O}\right)_{n}\right]^{-}, X$, and $X^{-}$species, respectively. The total energies $E_{n}$ and $E_{n}^{-}$can be expressed by the following energy partition:

$$
E_{n}=E_{\mathrm{int}\left[X\left(\mathrm{H}_{2} \mathrm{O}\right)_{n}\right]}+n E_{\mathrm{H}_{2} \mathrm{O}}+E
$$

and

$$
E_{n}^{-}=E_{\mathrm{int}\left[X\left(\mathrm{H}_{2} \mathrm{O}\right)_{n}\right]^{-}}+n E_{\mathrm{H}_{2} \mathrm{O}}+E^{-} .
$$

$E_{\mathrm{int}\left[X\left(\mathrm{H}_{2} \mathrm{O}\right)_{n}\right]}$ and $E_{\mathrm{int}\left[X\left(\mathrm{H}_{2} \mathrm{O}\right)_{n}\right]^{-}}$being the interaction energy of $\left[X\left(\mathrm{H}_{2} \mathrm{O}\right)_{n}\right]$ and $\left[X\left(\mathrm{H}_{2} \mathrm{O}\right)_{n}\right]^{-}$clusters, respectively, and $n E_{\mathrm{H}_{2} \mathrm{O}}$ is the energy of the $n$ water molecules. Substituting the previous definitions into Eq. (1), $E_{\text {stab }}(n)$ can be defined as

$$
E_{\text {stab }}(n)=E_{\text {int }\left[X\left(\mathrm{H}_{2} \mathrm{O}\right)_{n}\right]}-E_{\mathrm{int}\left[X\left(\mathrm{H}_{2} \mathrm{O}\right)_{n}\right]^{-}} .
$$

Within the statistical framework, as that adopted in this work, the consideration of the expected values for $E_{\text {int }}$ leads to

$$
\begin{aligned}
E_{\text {stab }}(n) & =\left\langle B E_{v}(n)-E A\right\rangle \\
& =\left\langle E_{\operatorname{int}\left[X\left(\mathrm{H}_{2} \mathrm{O}\right)_{n}\right]}-E_{\mathrm{int}\left[X\left(\mathrm{H}_{2} \mathrm{O}\right)_{n}\right]^{-}}\right\rangle .
\end{aligned}
$$

$E_{\text {stab }}(n)$ calculations at the temperatures previously cited were performed for all the structures saved along the MC simulation corresponding to a cluster of a given number of water molecules.

\section{RESULTS AND DISCUSSION}

Table I shows the calculated and experimental $E_{\text {stab }}(n)$ along with associated error for $\left[X\left(\mathrm{H}_{2} \mathrm{O}\right)_{n}\right]^{-}$clusters with $n$ $\leqslant 4$ at $200 \mathrm{~K}$. A good agreement is found between both theoretical estimations (average deviation around 5\%). In the case of the bromide and iodide anions a similar average deviation is found comparing theoretical and experimental results. On the contrary, significant differences $(\sim 20 \%)$ are observed for the fluoride-water clusters. This fact will be treated in detail below. The results obtained do not change when the number of structures considered from MC simulations was increased by saving every $0.1 M$ configurations instead of every $1 M$.

The agreement found between Koopmans and experimental estimations validates the assumptions used in computing the $E_{\text {stab }}(n)$ from first-principles interaction potentials, that is, the repolarization of the cluster during the photodetachment process is negligible. Likewise, the small differences between computed stabilization energies for the small clusters gives confidence in the use of MCDHO estimations in the case of larger clusters that are too computationally demanding for $a b$ initio calculations.

The structural analysis of the cluster geometries employed in Table I shows the lack of a direct relation between the value of $E_{\text {stab }}$ and the relative halide ion-water positions in the cluster. This is illustrated in Fig. 1 which shows how clusters having different structures present similar values of $E_{\text {stab }}(n)$ and vice versa. What is more, there is no connection between the interaction energy of the ionic cluster (given in

$\left[\mathrm{F}\left(\mathrm{H}_{2} \mathrm{O}\right)_{3}\right]^{-}$at $200 \mathrm{~K}$

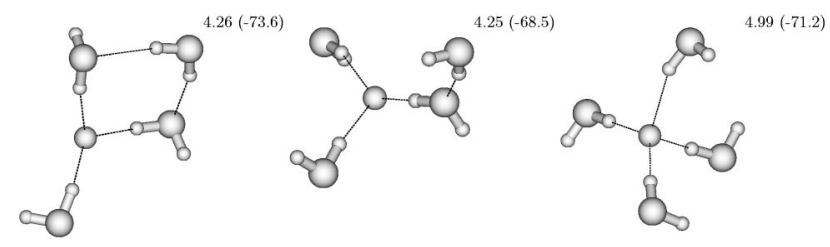

$\left[\mathrm{Br}\left(\mathrm{H}_{2} \mathrm{O}\right)_{3}\right]^{-}$at $200 \mathrm{~K}$
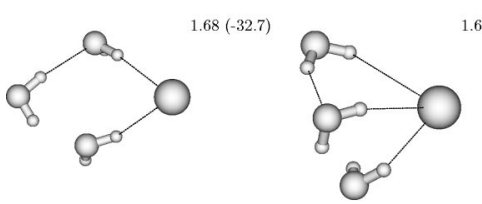

$1.66(-34.3)$

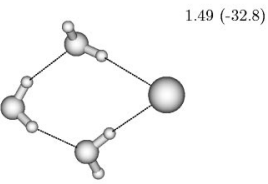

$\left[\mathrm{I}\left(\mathrm{H}_{2} \mathrm{O}\right)_{3}\right]^{-}$at $200 \mathrm{~K}$

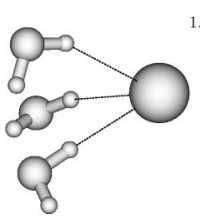

$1.26(-30.2)$

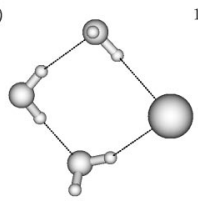

$1.14(-29.4)$

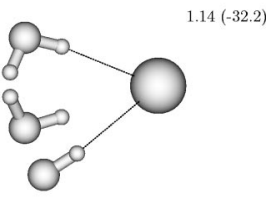

FIG. 1. $\left[X\left(\mathrm{H}_{2} \mathrm{O}\right)_{n}\right]^{-}$clusters derived from MC simulations at $200 \mathrm{~K}$ with their MCDHO predicted $E_{\text {stab }}(n)(\mathrm{eV})$ and MCDHO interaction energy $(\mathrm{kcal} / \mathrm{mol})$ in parenthesis. 


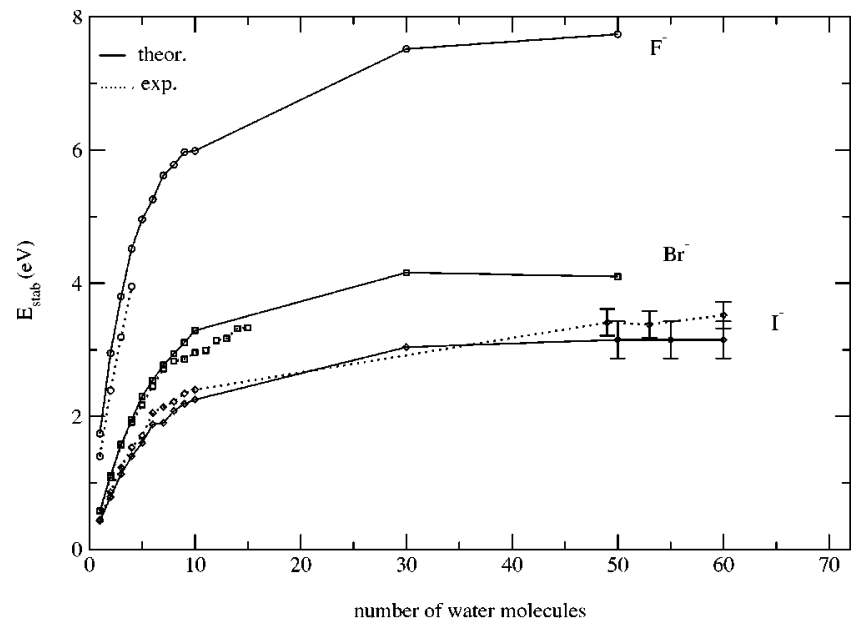

FIG. 2. Comparison of the MCDHO predicted (solid line) and experimental (dashed line) $E_{\text {stab }}(n)$ for $\left[X\left(\mathrm{H}_{2} \mathrm{O}\right)_{n}\right]^{-}$clusters $(X \equiv \mathrm{F}, \mathrm{Br}$, and I for $n$ $=1-60)$ at $200 \mathrm{~K}$. The uncertainty for $\left[\mathrm{I}\left(\mathrm{H}_{2} \mathrm{O}\right)_{(n \geqslant 50)}\right]^{-}$has been included.

parenthesis in Fig. 1) and $E_{\text {stab }}$, for example, $\left[\mathrm{Br}\left(\mathrm{H}_{2} \mathrm{O}\right)_{3}\right]^{-}$ clusters in Fig. 1 with almost the same interaction energies $(-32.7$ and $-32.8 \mathrm{kcal} / \mathrm{mol})$ have different $E_{\text {stab }}(3)(1.68$ and $1.49 \mathrm{eV})$. Similar examples are found for the other halides. Thus, it is not possible to discriminate among different structures only based on this property. Our conclusions on this matter are partly opposite to those presented by Combariza et al. ${ }^{24,25}$ who find evidences about a prevalent isomer of $\left[X\left(\mathrm{H}_{2} \mathrm{O}\right)_{n}\right]^{-}$clusters comparing the experimental $E_{\text {stab }}(n)$ and those obtained from $a b$ initio calculations on minimum structures. Nevertheless, these authors work on minima structures at $0 \mathrm{~K}$ and limit their study only to a few conformers. It is interesting to consider that in the case of halide microhydration many of the optimized structures have similar energies and the order of stability can change when thermal corrections and zero point vibrational effects are included. ${ }^{17,19,20,37-39}$ Thus, it could be unsafe trying to understand the structure of $\left[X\left(\mathrm{H}_{2} \mathrm{O}\right)_{n}\right]^{-}$clusters in terms of local minima. In this sense, the analysis of our MC trajectories indicates how different structures with similar interaction energy present different $E_{\text {stab }}$ values. Therefore, the consideration of a large number of structures seems to be mandatory. In a rather different framework, such as the computation of $\mathrm{X}$-ray absorption spectra of bromide aqueous solutions, quite close conclusions are achieved. ${ }^{8}$

The experimental and computed $E_{\text {stab }}(n)$ using MCDHO interaction potentials at $200 \mathrm{~K}$ is plotted in Fig. 2. The agreement is fairly good, particularly if one bears in mind that no empirical information has been included in the development of the interaction potentials used and the same parameters, except the halide charge, have been considered for the ionic and neutral halide-water clusters. The trend observed along the halide group shows a decrease of the $E_{\text {stab }}(n)$ going from $\mathrm{F}^{-}$to $\mathrm{I}^{-}$. The analysis of this figure provides information of the size dependence on vertical ionization, a fact that has already been observed. ${ }^{14,15,23-25}$ The associated uncertainties of the $E_{\text {stab }}(n)$ for the larger $\left[\mathrm{I}\left(\mathrm{H}_{2} \mathrm{O}\right)_{n}\right]^{-}$clusters is included in Fig. 2. They show that the discrepancies between computed and experimental estimations are within the range of

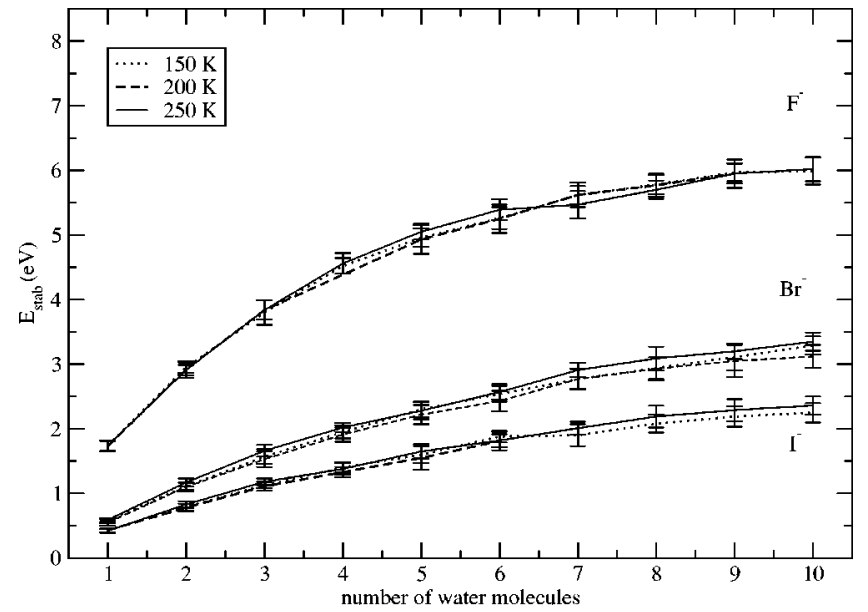

FIG. 3. $E_{\text {stab }}(n)$ for $\left[X\left(\mathrm{H}_{2} \mathrm{O}\right)_{n}\right]^{-}(X \equiv \mathrm{F}, \mathrm{Br}$, and I for $n=1-10)$ in $\mathrm{eV}$ at different temperatures and the uncertainty associated to each temperature.

these uncertainties. In addition, it should be taken into account that the experimental estimation of $E_{\text {stab }}(n)$ for larger clusters may be partially clouded by inelastic scattering of the emerging photoelectrons what may cause an increase of the magnitude. ${ }^{14}$

For clusters from 1 to 10 water molecules, Fig. 3 shows the $E_{\text {stab }}(n)$ values obtained at three different temperatures, 150,200 and $250 \mathrm{~K}$. Like previous works, ${ }^{26-29}$ our results are not temperature depending. Figure 3 also includes the uncertainty associated to the magnitude which increases when higher temperatures are considered. At $T=250 \mathrm{~K}$, the standard deviation associated to the $E_{\text {stab }}(n)$ estimation for $n \geqslant 6$ give rise to the overlap between $E_{\text {stab }}(n)$ and $E_{\text {stab }}(n$ $+1)$. This being particularly relevant for the case of iodide where the $E_{\text {stab }}$ gap between $n$ and $n+1$ is far smaller for $n \geqslant 6$. Markovich et al. ${ }^{14}$ postulate the completeness of the first hydration shell of the iodide ion for $n=6$ on the basis of the small difference found between $E_{\text {stab }}(6)$ and $E_{\text {stab }}(7)$. Although our results predict this small difference, they do not support the idea of a fully solvated iodide ion, because the structural analysis of the trajectory used to calculate $E_{\text {stab }}(6)$ indicates a preference of the structures where the iodide ion is on the surface of the cluster and some of the water molecules are located on the second solvation shell, as shown in Fig. 4, what also agrees with the results of Dang and Garret. ${ }^{26}$

A parallel study has been performed at $70 \mathrm{~K}$, but in this case a clear dependence on the initial configuration is found. At this temperature, the cluster is trapped into energy minima without being able to overcome the barrier and difficulting an appropriate sampling of the configurational space. In that

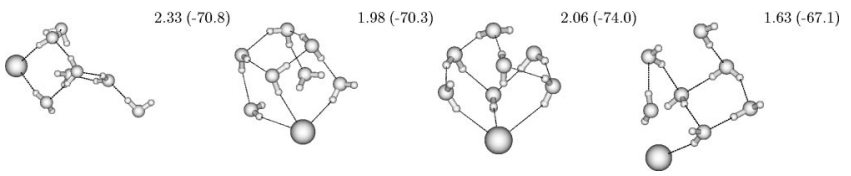

FIG. 4. $\left[\mathrm{I}\left(\mathrm{H}_{2} \mathrm{O}\right)_{6}\right]^{-}$clusters derived from MC simulations at $200 \mathrm{~K}$ with their MCDHO predicted $E_{\text {stab }}(n)(\mathrm{eV})$ and MCDHO interaction energy $(\mathrm{kcal} / \mathrm{mol})$ in parenthesis. 
TABLE II. Average stabilization energy $\left\langle E_{\text {stab }}(n)\right\rangle$ as a function of temperature using parallel tempering.

\begin{tabular}{rcrcc}
\hline \hline \multicolumn{2}{c}{$\left.\left[\mathrm{I}_{2} \mathrm{H}_{2} \mathrm{O}\right)_{8}\right]^{-}$} & & \multicolumn{2}{c}{$\left[\mathrm{I}_{\left.\left(\mathrm{H}_{2} \mathrm{O}\right)_{60}\right]^{-}}\right.$} \\
\cline { 1 - 2 } \cline { 5 - 5 }$T(\mathrm{~K})$ & $E_{\text {stab }}(8)(\mathrm{eV})$ & & $T(\mathrm{~K})$ & $E_{\text {stab }}(60)(\mathrm{eV})$ \\
\hline 60 & 2.25 & & 190 & 3.00 \\
70 & 2.24 & & 200 & 3.11 \\
90 & 2.22 & & 217 & 3.19 \\
110 & 2.20 & & 234 & 3.18 \\
130 & 2.17 & & 250 & 3.15 \\
150 & 2.15 & & & \\
170 & 2.12 & & & \\
\hline \hline
\end{tabular}

respect, $E_{\text {stab }}(8)$ for the iodide anion can be found equal to 2.4 or $2.0 \mathrm{eV}$ depending on the initial configuration used. Similar results to those obtained at $70 \mathrm{~K}$ have been found for larger aggregates $(n \geqslant 50)$ of the iodide anion at $T \leqslant 200 \mathrm{~K}$, that is, the final average value of $E_{\text {stab }}(n)$ is clearly biased by the starting point structure.

In order to ascertain if the variations of $E_{\text {stab }}$ estimations observed for clusters at $70 \mathrm{~K}$ and bigger clusters $(n \geqslant 50)$ of the iodide ion at $T \leqslant 200 \mathrm{~K}$ are only due to a sampling deficiency, we have used the parallel tempering ${ }^{40}$ (PT) method. This technique allows to overcome energy barriers that arise in the potential energy surfaces of the halide ion-water aggregates. In parallel tempering, a series of noninteracting replicas of the system under study is considered simultaneously. Each replica of the system can be viewed in a different state, characterized by some variables (e.g., temperature). The key of the PT is that in addition to conventional MC moves within each of the replicas, the identity of any two replicas is allowed to mutate. Supposing two replicas, $i$ and $j$, such a mutation is accepted with probability

$$
P_{a c c}=\min \left[1, \exp \left(-\Delta \beta_{i j} \Delta U_{i j}\right)\right],
$$

where $\Delta U_{i j}$ is the difference in energy between replicas $i$ and $j$ and $\Delta \beta_{i j}$ is the difference between their inverse temperature. As can be infered from Eq. (6), the acceptance rate for mutation moves will be sufficiently high only when the energy distribution functions for different states overlap significantly.

In the case of the small clusters at $70 \mathrm{~K}$ we have applied PT method to the $\left.\left[\mathrm{I}\left(\mathrm{H}_{2} \mathrm{O}\right)_{8}\right)\right]^{-}$aggregate that gives rise to two different $E_{\text {stab }}(8)$ estimations depending on the initial structure. Seven replicas (states) characterized by seven different temperatures $(60,70,90,110,130,150,170 \mathrm{~K})$ were considered. Configurations (200M) were run for each temperature trying mutations every 20000 steps. The $E_{\text {stab }}(8)$ estimations at each temperature are included in Table II and shows how $E_{\text {stab }}(8)$ at $70 \mathrm{~K}$ has a value in agreement with those at higher temperatures where ergodicity problems are not present.

An analysis of two trajectories of the $\left[\mathrm{I}\left(\mathrm{H}_{2} \mathrm{O}\right)_{60}\right]^{-}$aggregate at $200 \mathrm{~K}$, which result in different average value of $E_{\text {stab }}(n)(3.0$ vs $3.3 \mathrm{eV})$, indicates that the anion does not change its relative position in the clusters along the MC simulation. This analysis has been performed by plotting the distance between the iodide anion and the center of mass of the water molecules in the cluster along the trajectory. This

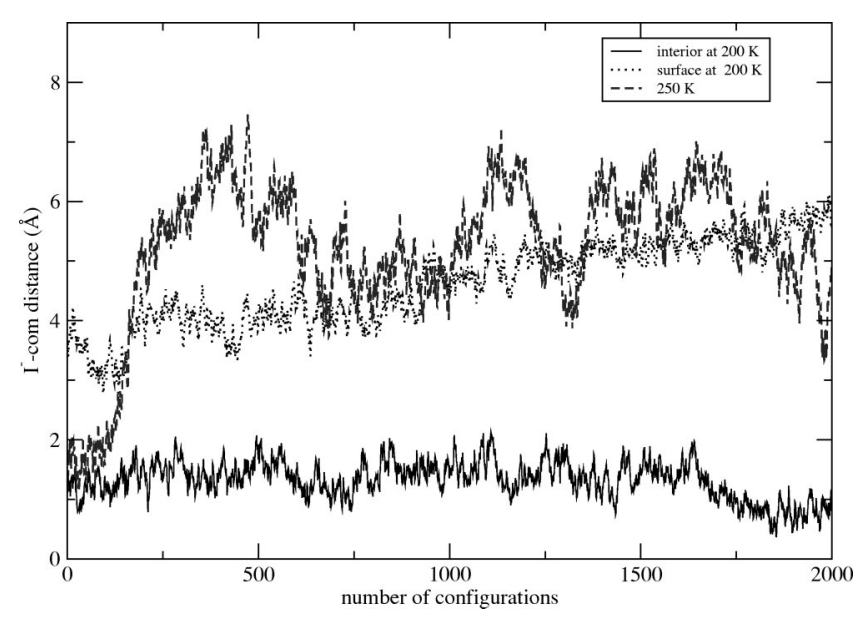

FIG. 5. Change of the distance $(\AA)$ between iodide ion and the center of mass of water molecules in the cluster for (a) an interior cluster at $200 \mathrm{~K}$, (b) a surface cluster at $200 \mathrm{~K}$ and (c) an interior cluster at $250 \mathrm{~K}$ along a Monte Carlo simulation.

plot is displayed in Fig. 5 and shows how the iodide ion stays approximately in the same place, i.e., if the anion is located on the surface of the cluster is not able to break the network of water molecules to penetrate inside the aggregate and vice versa. The situation is different at higher temperatures because the anion can move from the interior to the surface of the cluster without major problem. A PT MC simulation considering five different temperatures (190, 200, 217, 234, and $250 \mathrm{~K})$ were used to compute $E_{\text {stab }}(60)$ of the $\left.\left[\mathrm{I}\left(\mathrm{H}_{2} \mathrm{O}\right)_{60}\right)\right]^{-}$ cluster at $200 \mathrm{~K} .200 \mathrm{M}$ configurations were run for each temperature trying mutations every 10000 steps. The plot of the distance between the iodide anion and the center of mass of the water molecules in the cluster along the trajectory at $200 \mathrm{~K}$ using PT is showed in Fig. 6. This figure indicates how the anion changes its relative position in the cluster along the trajectory resulting in a $E_{\text {stab }}(60)$ estimation of 3.1 $\mathrm{eV}$. The results for the rest of temperatures are also included in Table II.

These two examples show that there is no dependence of $E_{\text {stab }}$ with the temperature at which the structures have been

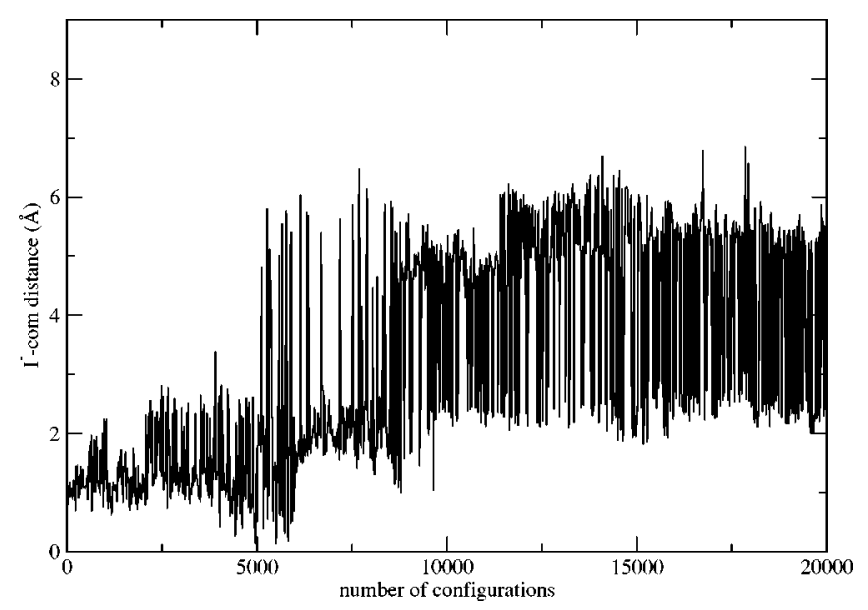

FIG. 6. Change of the distance $(\AA)$ between iodide ion and the center of mass of water molecules in the cluster at $200 \mathrm{~K}$ along a Monte Carlo simulation using PT method. 
TABLE III. Comparison among our predicted $E_{\text {estab }}(\mathrm{eV})$ estimations for $\left[\mathrm{Br}\left(\mathrm{H}_{2} \mathrm{O}\right)_{n}\right]^{-}$clusters with experimental and theoretical available data.

\begin{tabular}{rcccc}
\hline \hline & $\begin{array}{c}\text { This } \\
n\end{array}$ work $^{\mathrm{a}}(250 \mathrm{~K})$ & $\begin{array}{c}\text { Reference } 41^{\mathrm{b}} \\
(300 \mathrm{~K})\end{array}$ & $\begin{array}{c}\text { Reference } 41^{\mathrm{c}} \\
(300 \mathrm{~K})\end{array}$ & Expt. $^{\mathrm{d}}$ \\
\hline 1 & 0.57 & 0.60 & 0.55 & 0.57 \\
2 & 1.08 & 1.11 & 1.00 & 1.08 \\
3 & 1.43 & 1.59 & 1.42 & 1.58 \\
4 & 1.80 & 2.02 & 1.78 & 1.91 \\
5 & 2.22 & $2.41(275 \mathrm{~K})$ & $2.12(275 \mathrm{~K})$ & 2.17 \\
6 & 2.43 & $2.72(275 \mathrm{~K})$ & $2.37(275 \mathrm{~K})$ & 2.45 \\
7 & 2.77 & $2.98(275 \mathrm{~K})$ & $2.58(275 \mathrm{~K})$ & 2.71 \\
8 & 2.93 & $3.19(250 \mathrm{~K})$ & $2.78(250 \mathrm{~K})$ & 2.83 \\
9 & 3.05 & $3.41(250 \mathrm{~K})$ & $2.94(250 \mathrm{~K})$ & 2.86 \\
10 & 3.12 & $3.52(240 \mathrm{~K})$ & $3.06(240 \mathrm{~K})$ & 2.96 \\
\hline \hline
\end{tabular}

${ }^{\mathrm{a}} E_{\text {stab }}(n)$ computed using MCDHO interaction potentials.

${ }^{\mathrm{b}} \mathrm{SPC} / \mathrm{E}$. Pairwise additive interaction potential.

${ }^{\mathrm{c}} \mathrm{POL} 1$. Many-body effects are included in the model used.

${ }^{\mathrm{d}}$ Reference 42.

obtained, as long as the sampling of the potential energy surface is properly done.

Our MCDHO predicted results for $\left[\mathrm{Br}\left(\mathrm{H}_{2} \mathrm{O}\right)_{n}\right]^{-}$and $\left[\mathrm{I}\left(\mathrm{H}_{2} \mathrm{O}\right)_{n}\right]^{-}$are compared with experimental and previous computed values, ${ }^{27,41}$ in Tables III and IV. The agreement found with the experimental data is very good.

Figure 7 shows the $E_{\text {stab }}(n)$ estimations normalized to $E_{\text {stab }}(1)\left[E_{\text {stab }}(n) / E_{\text {stab }}(1)\right]$ for $\mathrm{F}^{-}, \mathrm{Br}^{-}$, and $\mathrm{I}^{-}$. This transformation, suggested by Perera and Berkowitz, ${ }^{28}$ allows the comparison of the property along the group easily. The experimental results (at the top of Fig. 7) show a different behavior of the fluoride ion in comparison with the bromide and iodide ones. Nevertheless, the absence of data for larger clusters in the case of the fluoride ion precludes an extensive comparison of the different behavior among the halide clusters. The computed theoretical estimations show (at the bottom of Fig. 7) the same tendency and confirm the different quantitative behavior of the fluoride anion. This fact has already been observed by us in a previous study of the structural properties of the halide solvation, ${ }^{5,6}$ where the fluoride ion differs from the rest of the group.

The agreement found between experimental and theoretical results improves when the normalization transformation is done. What is more, Figure 8 shows how the discrepancies observed among Koopmans, MCDHO, and experimental es-

TABLE IV. Comparison among our predicted $E_{\text {estab }}(\mathrm{eV})$ estimations for $\left[\mathrm{I}\left(\mathrm{H}_{2} \mathrm{O}\right)_{n}\right]^{-}$clusters with experimental and theoretical available data.

\begin{tabular}{ccccc}
\hline \hline & $\begin{array}{c}\text { This } \\
\text { work }^{\mathrm{a}}\end{array}$ & Reference $27^{\mathrm{b}}$ & Reference $27^{\mathrm{c}}$ & Expt. $^{\mathrm{d}}$ \\
\hline 1 & 0.42 & 0.47 & 0.44 & 0.45 \\
2 & 0.83 & 0.92 & 0.87 & 0.86 \\
3 & 1.18 & 1.41 & 1.32 & 1.23 \\
4 & 1.38 & 1.83 & 1.72 & 1.53 \\
5 & 1.65 & 2.11 & 2.05 & 1.71 \\
6 & 1.82 & 2.42 & 2.34 & 2.05 \\
\hline \hline
\end{tabular}

${ }^{a}$ At $150 \mathrm{~K}$ using MCDHO interaction potentials.

${ }^{\mathrm{b}}$ Classical simulation at $70 \mathrm{~K}$.

${ }^{c}$ Quantum simulation at $70 \mathrm{~K}$.

${ }^{\mathrm{d}}$ Reference 42.

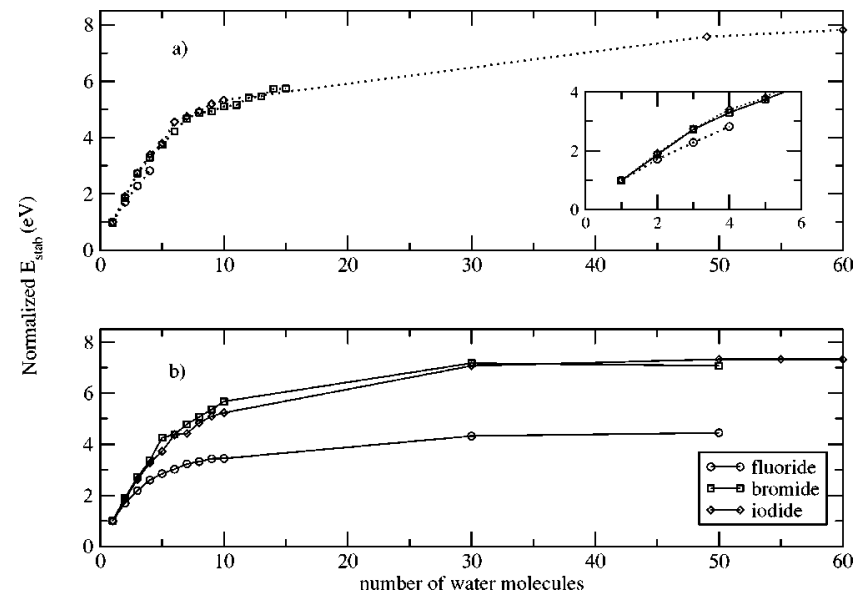

FIG. 7. (a) Plot of the normalized experimental $E_{\text {stab }}(n)$ for $\left[X\left(\mathrm{H}_{2} \mathrm{O}\right)_{n}\right]^{-}$vs the number of water molecules in the cluster. (b) Plot of the normalized MCDHO predicted $E_{\text {stab }}(n)$ for $\left[X\left(\mathrm{H}_{2} \mathrm{O}\right)_{n}\right]^{-}$vs the number of water molecules in the cluster.

timations of the fluoride-water clusters are diminished to a great extent. This seems to suggest that there would be an offset in the predicted values of the fluoride-water clusters that once corrected for $n=1$ would not appear again for larger $n$ values.

\section{CONCLUDING REMARKS}

We have predicted the stabilization energies of $\left[X\left(\mathrm{H}_{2} \mathrm{O}\right)_{n}\right]^{-}$clusters coming from MC simulations at different temperatures using first-principles $a b$ initio potentials. The good agreement between our predicted $E_{\text {stab }}(n)$ and experimental data allows us to validate the first-principles interaction potentials developed elsewhere and used in this study. ${ }^{5,6}$ It is particularly significant that this agreement was achieved without any specific parametrization of the interaction potentials to fit the magnitude under study. The fact that the difference between vertical photoionization of the halide clusters could be pretty well predicted by the cancellation of the net charge in the classical MCDHO interaction potential is a clear evidence of the electrostatic control of the $E_{\text {stab }}(n)$

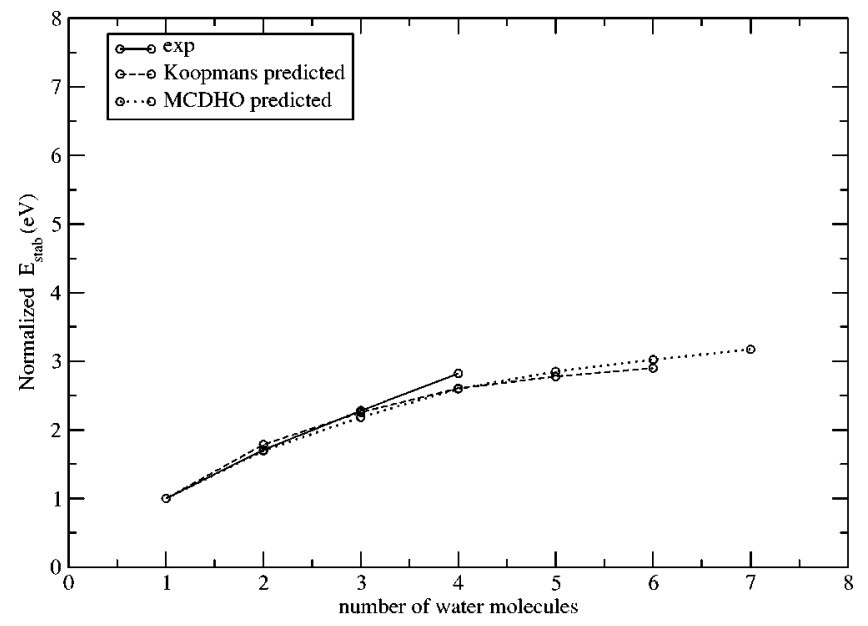

FIG. 8. Comparison of the normalized Koopmans, MCDHO, and experimental $E_{\text {stab }}(n)$ for $\left[\mathrm{F}\left(\mathrm{H}_{2} \mathrm{O}\right)_{n}\right]^{-}$clusters. 
magnitude. This should not be too surprising if one recognizes that the magnitude examined is the difference between the quantum mechanical property of the vertical ionization potential for two intimately related systems, $X^{-}$and $\left.\left[X\left(\mathrm{H}_{2} \mathrm{O}\right)_{n}\right)\right]^{-}$.

Results show that $E_{\text {stab }}(n)$ increases with the number of solvent molecules asymptotically to the value for infinite dilute solution.

Although there is no important influence of temperature on the $E_{\text {stab }}$ estimations, the uncertainty associated increases with it. The relevant aspect associated to temperature is the limited efficiency for sampling the configurational space of some of these systems when low temperature are considered, which can be solved by alternative approches such as PT method.

A clear relationship between the value of $E_{\text {stab }}(n)$ and the kind of structure that clusters present has not been found. Besides, the decreasing of the difference $E_{\text {stab }}(n)-E_{\text {stab }}(n$ -1) cannot be taken as criterion to identify when the halide ion is fully solvated, especially, in the case of the iodide anion where surface structures are dominant.

The diversity of $E_{\text {stab }}(n)$ values found for different clusters with similar stability indicates the need for statistical information to properly understand the stabilization energies of the halide anions.

$E_{\text {stab }}(n)$ decreases from $\mathrm{F}^{-}$to $\mathrm{I}^{-}$clusters. The discrepancies found between the fluoride, and bromide and iodide anions stresses the differential behavior that the former halide presents along its group.

Despite the fact that both theoretical estimations (Koopmans and $\mathrm{MCDHO}$ ) are adequate to reproduce experimental $E_{\text {stab }}$ of $\left[X\left(\mathrm{H}_{2} \mathrm{O}\right)_{n}\right]^{-}$clusters, the use of MCDHO firstprinciples interaction potentials supplys interesting adventages: it is less computationally demanding and can give access to larger clusters that could not be treated with ab initio methods.

\section{ACKNOWLEDGMENTS}

The authors wish to thank O. Cheshnovsky for useful explanations about the experimental data. This work was supported by Spanish DGICYT (Grant No. BQU200202217).

${ }^{1}$ A. Castleman, Jr. and K. Bowen, Jr., J. Phys. Chem. 100, 12911 (1996).

${ }^{2}$ A. Viggiano and S. Arnold, Int. Rev. Phys. Chem. 17, 147 (1998).

${ }^{3}$ M. P. Allen and D. J. Tildesley, Computer Simulation of Liquids (Oxford University Press, Oxford, 1987).

${ }^{4}$ D. W. Hermann, Computer Simulation Methods (Springer, Berlin, 1990).

${ }^{5}$ R. Ayala, J. M. Martínez, R. R. Pappalardo, H. Saint-Martin, I. OrtegaBlake, and E. Sánchez Marcos, J. Chem. Phys. 117, 10512 (2002).
${ }^{6}$ R. Ayala, J. Martínez, R. Pappalardo, and E. Sánchez Marcos, J. Chem. Phys. 119, 9538 (2003).

${ }^{7}$ H. Saint-Martin, J. Hernandez-Cobos, M. I. Bernal-Uruchurtu, I. OrtegaBlake, and H. J. C. Berendsen, J. Chem. Phys. 113, 10899 (2000).

${ }^{8}$ P. J. Merkling, R. Ayala, J. Martínez, R. Pappalardo, and E. Sánchez Marcos, J. Chem. Phys. 119, 6647 (2003).

${ }^{9}$ G. Leopold, J. Ho, and W. Leneberger, J. Chem. Phys. 86, 1715 (1987).

${ }^{10}$ J. Ho, K. Ervin, and W. Leneberger, J. Chem. Phys. 93, 6987 (1991).

${ }^{11}$ G. Ganterfor, K. Meiwesbroer, and H. Lutz, Phys. Rev. A 37, 2716 (1988).

${ }^{12}$ G. Ganterfor, K. Meiwesbroer, and H. Lutz, Faraday Discuss. Chem. Soc. 88, 16 (1988).

${ }^{13}$ O. Cheshnovsky, K. Taylor, J. Conceiao, and R. Smalley, Phys. Rev. Lett. 64, 1785 (1990)

${ }^{14}$ G. Markovich, S. Pollac, R. Giniger, and O. Cheshnovsky, J. Chem. Phys. 101, 9344 (1994).

${ }^{15}$ G. Markovich, S. Pollack, R. Giniger, and O. Chesnovsky, Z. Phys. D: At., Mol. Clusters 26, 98 (1993).

${ }^{16}$ X. Yang, X.-B. Wang, and L.-S. Wang, J. Chem. Phys. 115, 2889 (2001).

${ }^{17}$ J. Baik, J. Kim, D. Majumdar, and K. S. Kim, J. Chem. Phys. 110, 9116 (1999).

${ }^{18}$ D. Majumdar, J. Kim, and K. S. Kim, J. Chem. Phys. 112, 101 (2000).

${ }^{19}$ J. Kim, H. M. Lee, S. S. Suh, and K. S. Kim, J. Chem. Phys. 113, 5259 (2000).

${ }^{20}$ H. M. Lee, D. Kim, and K. S. Kim, J. Chem. Phys. 116, 5509 (2002).

${ }^{21}$ H. M. Lee and K. S. Kim, J. Chem. Phys. 114, 4461 (2001).

${ }^{22}$ T. A. Koopmans, Physica (Amsterdam) 1, 104 (1933).

${ }^{23}$ J. E. Combariza, N. R. Kestner, and J. Jortner, Chem. Phys. Lett. 203, 423 (1993).

${ }^{24}$ J. E. Combariza, N. R. Kestner, and J. Jortner, J. Chem. Phys. 100, 2851 (1994).

${ }^{25}$ J. E. Combariza, N. R. Kestner, and J. Jortner, Chem. Phys. Lett. 221, 156 (1994).

${ }^{26}$ L. X. Dang and B. C. Garret, J. Chem. Phys. 99, 2972 (1993).

${ }^{27}$ H. Gai, G. K. Schenter, L. X. Dang, and B. C. Garret, J. Chem. Phys. 105, 8835 (1996).

${ }^{28}$ L. Perera and M. L. Berkowitz, J. Chem. Phys. 99, 4222 (1993).

${ }^{29}$ L. S. Sremaniak, L. Perera, and M. L. Berkowitz, Chem. Phys. Lett. 218, 377 (1994).

${ }^{30}$ T. H. Dunning, J. Chem. Phys. 90, 1007 (1989).

${ }^{31}$ R. A. Kendall, T. H. Dunning, and R. J. Harrison, J. Chem. Phys. 96, 6796 (1992).

${ }^{32}$ E. R. Davidson, Chem. Phys. Lett. 260, 51 (1996).

${ }^{33}$ C. W. Bauschlicher, Jr., H. F. Schaefer III, and P. S. Bagus, J. Am. Chem. Soc. 99, 7106 (1977).

${ }^{34}$ M. Kaupp, P. v. R. Schleyer, H. Stoll, and H. Preuss, J. Am. Chem. Soc. 113, 6012 (1991).

${ }^{35}$ A. Begner, M. Dolg, W. Kÿchle, H. Stoll, and H. Preuss, Mol. Phys. 80, 1431 (1993)

${ }^{36}$ J. M. L. Martin and A. Sundermann, J. Chem. Phys. 114, 3408 (2001).

${ }^{37}$ J.-H. Choi, K. T. Kuwata, Y.-B. Cao, and M. Okumura, J. Phys. Chem. A 102, 503 (1998)

${ }^{38}$ S. J. Vaughn, E. V. Akhmastkaya, M. A. Vincent, A. J. Masters, and I. H. Hillier, J. Chem. Phys. 110, 4338 (1999).

${ }^{39}$ R. A. Bryce, M. A. Vincent, and I. H. Hillier, J. Phys. Chem. A 103, 4094 (1999).

${ }^{40}$ D. Frenkel and B. Smit, Understanding Molecular Simulations: From Algorihms to Applications, 2nd ed. (Academic, London, 2002).

${ }^{41}$ L. S. Sremaniak, L. Perera, and M. L. Berkowitz, J. Phys. Chem. 100, 1350 (1996).

${ }^{42} \mathrm{O}$. Cheshnovsky (private communication). 